21st Particles and Nuclei International Conference (PANIC 2017)

International Journal of Modern Physics: Conference Series

Vol. 46 (2018) 1860027 (6 pages)

(C) The Author(s)

DOI: $10.1142 / \mathrm{S} 2010194518600273$

\title{
Hadron Form Factors at BESIII
}

\author{
Zhaoxia Meng (on behalf of BESIII Collaboration) \\ University of Jinan, 336 Nanxinzhuang west road \\ Jinan, Shandong Province 250022, China \\ sps_mengzx@ujn.edu.cn
}

Published 3 May 2018

\begin{abstract}
Form factors of hadron provide fundamental information about its structure and dynamics. They constitute a rigorous test of non-perturbative QCD as well as of phenomenological models. Based on data samples collected with BESIII detector at BEPCII collider, born cross section of $e^{+} e^{-} \rightarrow p \bar{p}$ and proton effective form factors are measured at 12 center-of-mass energies between 2.2324 and $3.671 \mathrm{GeV}$. The ratio $\left|G_{E} / G_{M}\right| \mathrm{s}$ are extracted by fitting polar angle distribution of proton for data samples with large statistics. For data between 3.773 and $4.6 \mathrm{GeV}$, we use initial state radiation (ISR) method to study $e^{+} e^{-} \rightarrow p \bar{p}$ by tagged or un-tagged ISR photon, where the pair cross section, effective form factors and $\left|G_{E} / G_{M}\right| \mathrm{s}$ are obtained from proton pair threshold to about $3 \mathrm{GeV}$. For $e^{+} e^{-} \rightarrow \Lambda \bar{\Lambda}$ and $e^{+} e^{-} \rightarrow \Lambda_{c}^{+} \bar{\Lambda}_{c}^{-}$, the pair cross section and $\Lambda / \Lambda_{c}$ form factors are measured near threshold. With data scanned in 2015 from 2-3.08 GeV, charged Kaon pair cross section and form factors are measured at 21 center-of-mass energies.
\end{abstract}

Keywords: BEPCII; BESIII; Hadron Form Factor.

\section{Introduction}

Hadrons have internal structures. Looking into hadron internal structures allows physicists to understand non-perturbative QCD. Form Factors (FFs) are the variables describing hadron internal structures, and can be measured in experiments ${ }^{1}$. Further more, FF at hadron production threshold provides important information to deeply understand the strong interaction.

This paper is going to summarize the results on hadron form factor measurements at BESIII. The facility and BESIII detector will be described in section 2 . Hadronic form factor measurements will be illustrated in section 3 .

\section{The facility and detector}

The BEijing Spectrometer (BESIII) ${ }^{2}$ is a general purpose detector installed at BEPCII collider ${ }^{2}$. BEPCII provides electron and positron beams with energy from

This is an Open Access article published by World Scientific Publishing Company. It is distributed under the terms of the Creative Commons Attribution 4.0 (CC-BY) License. Further distribution of this work is permitted, provided the original work is properly cited. 
1 to $2.3 \mathrm{GeV}$. Based on the data collected in the energy range, physics related to QCD, charmonium, charm, light hadrons and new physics can be studied. The BESIII detector is composed of the following components from inner to outer: 1). MDC (Multilayer Drift Chamber) measures momentum and energy loss $d E / d x$ of charged tracks; 2). TOF (Time Of Flight) provides a $2 \sigma \pi / K$ separation up to $1 \mathrm{GeV}$. Combining with $d E / d x$, it constitutes one of the essential variables in particle identification; 3). EMC (ElectroMagnetic Calorimeter) measures energy of photons and electrons, and also provide important information in electron and hadron separation; 4.) Superconductor provides a magnetic field of $1 \mathrm{~T}$; 5.) MUC (Muon Counter) is mainly used to identify muons especially for low momentum ones.

\section{Hadron form factors}

Normally, FFs are measured in elastic or annihilating process. In the former case (Space-Like (SL) region), electrons are provided as probers and targets are normally protons or neutrons. It is difficult to get unstable targets like $\Lambda$ or $\Sigma$. In the later case, called the Time-Like (TL) region, electron-positron annihilate into hadrons that may be stable or unstable. BESIII is in the TL region. In both cases, as shown in Fig. 1, two identical interacting vertices are involved for the first leading order diagram. One of the vertices involves only electromagnetic interactions which can be calculated precisely with QED. Another vertex where hadrons are not proper to be treated as point-like particles.

\subsection{Baryon form factors}

For baryons with spin $\frac{1}{2}$, the vertex related to hadrons can be described into Dirac and Pauli FFs, displayed in eq. (1).

$$
\Gamma_{\mu}=\gamma_{\mu} F_{1}\left(q^{2}\right)+\frac{i \sigma_{\mu \nu} q^{\nu}}{2 M_{B}} \kappa F_{2}\left(q^{2}\right)
$$

where $F_{1}\left(q^{2}\right)$ and $F_{2}\left(q^{2}\right)$ are Dirac and Pauli form factor respectively, $q^{2}=s=$ $\left(k+k^{\prime}\right)^{2}$ is negative for SL region and positive for TL region, $\kappa$ is the anomalous magnetic moment of the baryon.

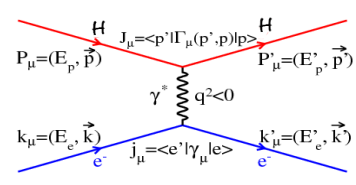

(a)

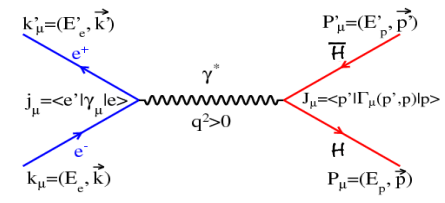

(b)

Fig. 1. The Feynman diagrams for baryon production in SL region (a) and TL region (b). 
The Sachs FFs, $G_{E}$ and $G_{M}$ are linear function of Dirac and Pauli FFs. They are defined as in eq. (2), where $\tau=\frac{q^{2}}{4 M_{B}^{2}}$.

$$
G_{E}\left(q^{2}\right)=F_{1}\left(q^{2}\right)+\tau \kappa F_{2}\left(q^{2}\right), \quad G_{M}\left(q^{2}\right)=F_{1}\left(q^{2}\right)+\kappa F_{2}\left(q^{2}\right) .
$$

The $G_{E}$ and $G_{M}$ are also named as electromagnetic FFs (EMFFs), illustrating the electric and magnetic charge distribution of the baryon ${ }^{3}$. They are normally measured through angular analysis. In the TL region, the differential cross section of baryon production can be expressed in terms of $G_{E}$ and $G_{M}$, displayed in eq. (3).

$$
\frac{d \sigma_{b o r n}}{d \Omega}\left(q^{2}, \theta\right)=\frac{\alpha^{2} \beta C}{4 q^{2}}\left[\frac{1}{\tau} \sin ^{2} \theta\left|G_{E}\right|^{2}+\left(1+\cos ^{2} \theta\right)\left|G_{M}\right|^{2}\right] .
$$

where $\theta$ is the polar angle between the out going baryons and beam line, $\mathrm{C}$ is the Coulomb correction and $\beta=\sqrt{1-1 / \tau}$.

The born cross section is then expressed in eq. (4). If $|G|=\left|G_{E}\right|=\left|G_{M}\right|$ is assumed, effective FF $G$ can be obtained by measuring the born cross section, as shown in eq. (5).

$$
\begin{gathered}
\sigma_{b o r n}=\frac{4 \pi \alpha^{2} \beta C}{3 q^{2}}\left[\left|G_{M}\right|^{2}+\frac{1}{2 \tau}\left|G_{E}\right|^{2}\right] . \\
\left|G\left(q^{2}\right)\right|=\sqrt{\sigma_{b o r n} /\left[\frac{4 \pi \alpha^{2} \beta C}{3 q^{2}}\left(1+\frac{1}{2 \tau}\right)\right]} .
\end{gathered}
$$

Two methods have been used at BESIII to measure the proton FFs: data scan ${ }^{4}$ and ISR process.

With data scanned in 2011 and 2012, proton pair production have been studied at 12 energy points, which is shown in Fig. 2. Two opposite charged tracks are required to be generated from the interaction point (IP) that they both pass within $1 \mathrm{~cm}$ in the transverse plane and $10 \mathrm{~cm}$ along the beam. Particle identification (PID) probability is calculated using combined information of $d E / d x$ and TOF. It is tagged as a proton (anti-proton) if the highest probability assigned to the track is a proton (anti-proton). $E / p<0.5$ is required for each proton (anti-proton) candidate to suppress the background from Bhabha. The absolute difference of the time of flight in the TOF between the two tracks is required to be smaller than $4 \mathrm{~ns}$ to suppress the cosmic background. For samples with center-of-mass energy larger than $2.4 \mathrm{GeV}$, the polar angle of the proton is required to satisfy $|\cos \theta<0.8|$ to further suppress the Bhabha background. The opening angle between proton and anti-proton is required to be $\theta_{p \bar{p}}>1.78$ for $\sqrt{s} \leq 2.4 G e V$ and $\theta_{p \bar{p}}>1.79$ for $\sqrt{s}>2.4 \mathrm{GeV}$. Finally, $\left|p_{\text {mea }}-p_{\text {exp }}\right|<5 \sigma_{p}$ is required, where $p_{\text {mea }}$ and $p_{\text {exp }}$ are the measured and expected proton (anti-proton) momentum in the center-of-mass energy system, and $\sigma_{p}$ is the momentum resolution. The angular distributions are shown in Fig. 3 and the corresponding $\left|G_{E} / G_{M}\right|$ are shown in Fig. 4.

Preliminary results on form factors using ISR process have been obtained as well. The cross section and effective form factors are measured in 31 energy intervals 


\section{Z. Meng}

from proton pair production threshold to about $3 \mathrm{GeV}$. Due to statistic limitation, angular distribution and $\left|G_{E} / G_{M}\right|$ are measured in wider energy intervals. The results are consistent with previous experiments and BESIII using scanned data.
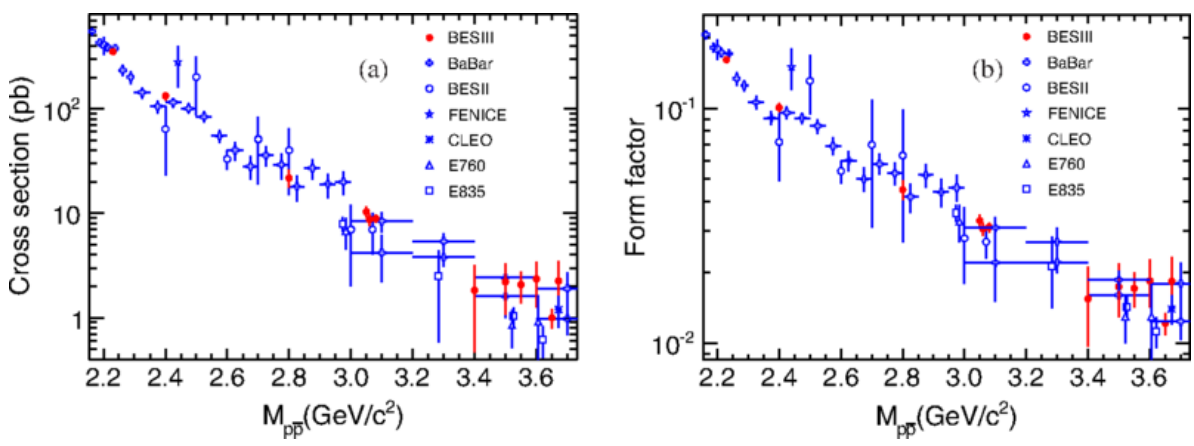

Fig. 2. The cross section of $e^{+} e^{-} \rightarrow p^{+} p^{-}$(a) and proton form factors (b) at 12 energy points. The results are compared to previous experiments and the uncertainty is improved by about $30 \%$ comparing to Babar.
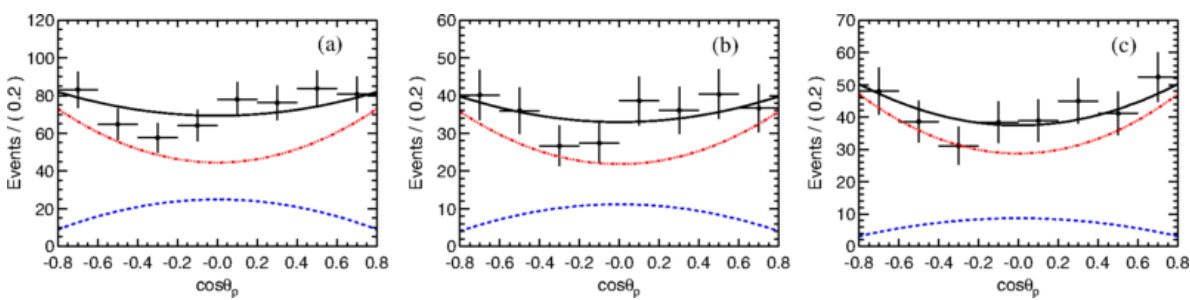

Fig. 3. The angular distribution proton/anti-proton at energies (a) $2.2324 \mathrm{GeV}$, (b) $2.4 \mathrm{GeV}$ and (c) combined 3.05, 3.06 and $3.08 \mathrm{GeV}$.

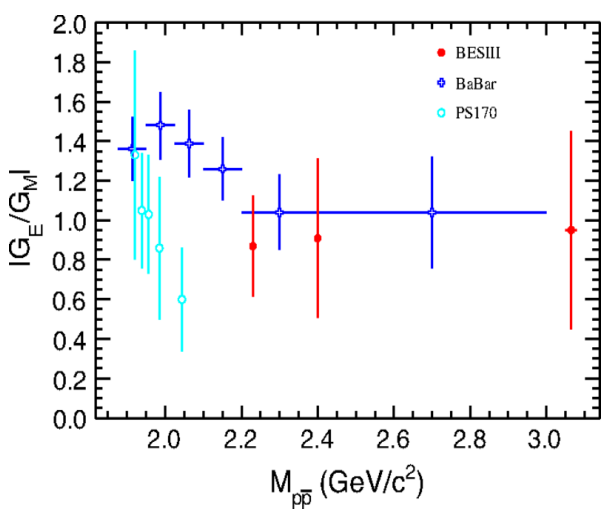

Fig. 4. The $\left|G_{E} / G_{M}\right|$ of proton at $2.2324 \mathrm{GeV}, 2.4 \mathrm{GeV}$ and combined $3.05,3.06$ and $3.08 \mathrm{GeV}$. The results are compared to previous experiments and consistent with Babar in the overlapped energy region. 
Preliminary results on $\Lambda / \bar{\Lambda}$ and $\Lambda_{c}^{+} / \bar{\Lambda}_{c}^{-}$have been obtained at BESIII. Fig. 5 (left) shows the effective form factor for $\Lambda / \bar{\Lambda}$. Jump at threshold is interesting and indicates that more considerations should be included in the theoretical calculation. To identify $e^{+} e^{-} \rightarrow \Lambda \bar{\Lambda}$ events, we use the decays $\Lambda \rightarrow p \pi^{-}$and $\bar{\Lambda} \rightarrow \bar{p} \pi^{+} / \bar{\Lambda} \rightarrow \bar{n} \pi^{0}\left(\bar{\Lambda} \rightarrow \bar{n} \pi^{0}\right.$ is used at $\left.2.2324 \mathrm{GeV}\right)$. At center-of-mass energy $2.2324 \mathrm{GeV}$, which is only about $1 \mathrm{MeV}$ above the $\Lambda / \bar{\Lambda}$ threshold, the momentum of the final particles are very low. It is almost impossible to observe proton tracks in MDC. Except identifying low momentum $\pi s$ by PID, the low momentum antiproton is identified through the secondary particles produced due to interacting with the beam pipe.

Fig. 5 (right) shows the $e^{+} e^{-} \rightarrow \Lambda_{c}^{+} \bar{\Lambda}_{c}^{-}$pair cross section. Different from Belle results, the cross section near by threshold dose not drop to very low value.

\subsection{Meson form factor}

Meson form factors are normally extracted according to eq. (6). Preliminary results on $e^{+} e^{-} \rightarrow K^{+} K^{-}$using data scanned in 2015 from 2 to $3.08 \mathrm{GeV}$ are shown in Fig. 6. The results are consistent with other experiments with much smaller uncertainty. A clear bump is seen around $2.2 \mathrm{GeV}$ in the line shape, which indicates more studies needed for further investigations.

$$
\left|F_{K}\right|^{2}\left(q^{2}\right)=\frac{3 q^{2}}{\pi \alpha(0)^{2} \beta_{K}^{2}} \frac{\sigma_{K K}\left(q^{2}\right)}{C_{F S}} .
$$

where $\sigma_{K K}$ is the dressed cross section, defined as $\sigma_{K K}^{0}\left(q^{2}\right)\left(\frac{\alpha\left(q^{2}\right)}{\alpha(0)}\right)^{2}$ with $\sigma_{K K}^{0}$ the bare cross section; $\beta=\sqrt{1-4 m_{K}^{2} / q^{2}} ; C_{F S}$ is the final-state correction.
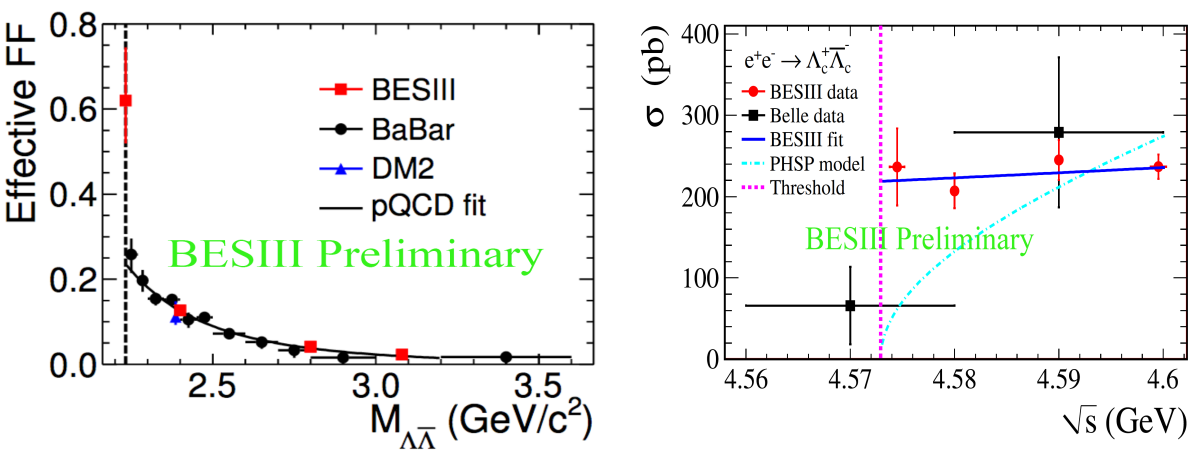

Fig. 5. Effective form factor of $e^{+} e^{-} \rightarrow \Lambda \bar{\Lambda}$ at 4 energy points $2.2324 \mathrm{GeV}, 2.4 \mathrm{GeV}, 2.8 \mathrm{GeV}$, $3.08 \mathrm{GeV}$, where $2.2324 \mathrm{GeV}$ is just $1 \mathrm{MeV}$ above the $\Lambda$ pair threshold (left). Cross section of $e^{+} e^{-} \rightarrow \Lambda_{c}^{+} \bar{\Lambda}_{c}^{-}$at energy points $4.5745 \mathrm{GeV}, 4.58 \mathrm{GeV}, 4.59 \mathrm{GeV}$ and $4.5995 \mathrm{GeV}$, where $4.5745 \mathrm{GeV}$ is $1.6 \mathrm{MeV}$ above the threshold (right). 

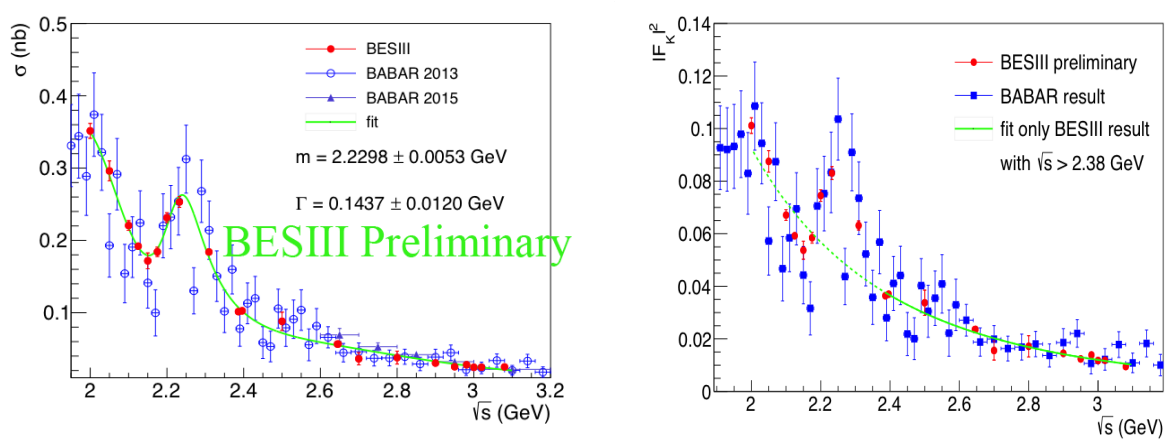

Fig. 6. Cross section (left) and effective form factor (right) of charge Kaon at BESIII (red points).

\section{Summary}

A plenteous measurements on hadron form factors have been performed at BESIII. With data collected in 2011 and 2012, proton form factors have been measured at 12 energy points ${ }^{4}$. With data collected from 2010 to 2014 , proton form factors have been measured using initial state radiation (ISR) process at 31 energy intervals from proton pair production threshold to $3 \mathrm{GeV}$. $\Lambda$ form factors have been measured at 4 energy points using data collected in 2015. $\Lambda_{c}$ form factors have been measured using data collected from 2011 to 2014. Besides, plenty of meson form factors have been measured at BESIII. Preliminary results on charged Kaon have been obtained. For pion and D meson form factors please refer to $[5,6]$. Measurements of neutron, $\Sigma, \Xi$ etc. are on going or in plan.

\section{Acknowledgments}

This work is supported partially by National Natural Science Foundation of China (NSFC) under Contract No. 11605074. The BESIII Collaboration thanks the staff of BEPCII and the computing center for their strong supports.

\section{References}

1. Gernot Eichmann, Progress in Particle and Nuclear Physics 67 (2012), p. 234.

2. BESIII Collaboration (M. Ablikim et al.), Nucl. Instrum. Meth. A 614 (2010), p. 345.

3. Johansson Tord, J. University of Science and Technology of China 46 (2016), 4.

4. BESIII Collaboration (M. Ablikim et al.), Phys.Rev. D 91 (2015) 11, 112004

5. BESIII Collaboration (M. Ablikim et al.), Phys.Lett. B 753 (2016), p. 629.

6. BESIII Collaboration (M. Ablikim et al.), Phys.Rev. D 92 (2015) 7, 071101 